\title{
INTRACUTANEOUS INFECTION WITH LEPTOSPIRA ICTEROHAEMORRHAGIAE (SHIBAURA STRAIN) OF THE GUINEA PIG
}

\author{
MAMORU MORI, YOSHIKO ARIMITSU, \\ SHO OTANI AND KIYOTO AKAMA \\ The 2nd Department of Bacteriology, National Institute of Health, \\ (WHO/FAO Leptospirosis Reference Laboratory), \\ Shinagawa-ku, Tokyo 141, Japan
}

(Received: July 13, 1974)

\begin{abstract}
SUMMARY : Experimental leptospirosis with Leptospira icterohaemorrhagiae Shibaura strain was studied in guinea pigs. When the pathogen was inoculated intracutaneously to the back of the animals, localized haemorrhage was observed at the inoculated site before the appearance of general haemorrhage. The severity of the local lesion increased progressively until the 7th day of inoculation. The minimum infective dose (MID) or the $50 \%$ infective dose $\left(\mathrm{ID}_{50}\right)$ of the leptospiral suspension was determined by the appearance of the macroscopic local haemorrhage 7 days after inoculation. The MID thus determined was almost comparable with the value determined by the development of general symptoms and signs by conventional ip inoculation. The number of the pathogen per $\mathrm{ID}_{50}$ varied between 6 and 35 in five experiments. The local haemorrhage was effectively protected by active or passive immunization.

Microscopically, haemorrhage at the inoculated site was found mainly in the dermis, directly beneath the epidermis in particular, and accompanied with leakage of the pathogen. The pathogen was also detected abunduntly in the thickened epidermal layer covering the inoculated area as well as in the epithelial matrix of hair-follicle, probably due to the proliferation of the pathogen at the site.
\end{abstract}

\section{INTRODUCTION}

Patients suffering from Weil's disease usually develop dermal petechiae as a part of general haemorrhage at the acute stage of the disease. Similar symptoms are observed in animals infected experimentally with L. icterohaemorrhagiae. Haemorrhage localized at infected site was sometimes recognized clinically (Kaneko and Okuda, 1915) and experimentally (Inada, 1951), but little attention has been called for. During the early study of experimental leptospirosis in the guinea pig, the authors noticed appearance of haemorrhage localized at the inoculated site before the development of general haemorrhage. Such local haemorrhage was particularly remarkable when a strain called "Shibaura" was used for inoculation into the guinea pig skin. Since the haemorrhagic lesion was clearly observable with the naked eye, it seemed possible to use the ic infection method for titration of the virulence of the pathogen.

In this paper, the course of the disease produced by ic injection of Shibaura strain and the practical method for titration of virulence of the pathogen are described.

守・有光佳子・大谷 昌・赤真清人（国立予防衛生研究所 細菌第二部） 


\section{MATERIALS AND METHODS}

Animals: Guinea pigs Hartley/F of various weight were used for ic infection. Albino rabbits of about $3 \mathrm{~kg}$ were also used for comparison.

Strain: L. icterohaemorrhagiae Shibaura strain was isolated from Rattus norvegicus at Shibaura slaughter house, Tokyo, in 1964. The strain has been maintained in guinea pigs and used for preparation and assay of vaccine and therapeutic serum for Weil's disease in Japan since 1965.

Leptospiral suspension for inoculation: The leptospiral suspension used for infection was prepared by emulsifying the liver of guinea pigs dying with typical symptoms 5 to 7 days after infection. A $10 \%$ emulsion of the liver in phosphate buffered saline of $\mathrm{pH} 7.3$ consisting of $1.2 \mathrm{~g} \mathrm{Na} \mathrm{HPO}_{4}, 0.7 \mathrm{~g} \mathrm{KH}_{2} \mathrm{PO}_{4}$ and $6.8 \mathrm{~g} \mathrm{NaCl}$ per liter (PBS) was centrifuged at $3,500 \mathrm{rpm}$ for $30 \mathrm{~min}$ to remove the debris of the tissue and the supernatant fluid was used as leptospiral suspension unless otherwise mentioned. The number of the organism in the supernatant fluid was usually on a level of $10^{6} / \mathrm{ml}$ when counted with a Petroff-Hausser's bacterial counter.

Procedure for intracutaneous inoculation: The hair over the dorsal area of the animal was clipped off. One-tenth milliliter of the leptospiral suspension diluted to an appropriate concentration in PBS was injected into the back skin. The animals were observed daily for development of local haemorrhage at the injection site as well as appearance of general symptoms such as decrease in body weight, pyrexia, jaundice and haemorrhages from the nose or the anus.

For titrating the virulence of the pathogen, the leptospiral suspension was diluted serially at intervals of 0.5 in logarithm, and $0.1 \mathrm{ml}$ of each dilution was injected into the back skin of each of at least three guinea pigs individually. The end point of local haemorrhage was defined as the highest dilution of the leptospiral suspension producing a visible lesion at the injected site 7 days after the inoculation, irrespective of the size or severity of the lesion.

Procedure for intraperitoneal inoculation: One-milliliter portion of each of the serial dilutions of the leptospiral suspension were injected intraperitoneally into a group of guinea pigs. The animals were observed daily for the general symptoms as described above.

Estimation of agglutinin titer: Agglutinin titers of the sera from the infected animals were estimated by Shüffner-Mochtar's microscopic agglutination-lysis method (Schüffner and Mochtar, 1927).

Staining of histological preparation: A part of the skin was removed 7 days after infection and subjected to histological examinations. The sections of the sample were stained with hematoxyrin-eosin and Levaditi's stainings.

\section{RESULTS}

\section{Difference in the Susceptibility to the Intracutaneous Infection between Guinea Pigs and Rabbits}

Patterns of development of local haemorrhage were compared in guinea pigs and rabbits. One-tenth milliliter each of serial dilutions of the suspension at intervals of 0.5 or 1.0 in logarithm was injected intracutaneously. In guinea pigs, local haemorrhage developed consistently even at very low doses of the leptospiral suspension. 
The intensity of the lesion increased progressively until the death of the animal which usually occurred between 5 to 7 days after inoculation. Necrosis or pus formation or both were sometimes observed in severe cases (Fig. 1). On autopsy, haemorrhage was recognized over the whole layer of the skin and often extended to the muscle layer just under the dermis.

In rabbits, six experiments were carried out with a total of 11 animals. The local haemorrhage was usually recognized on the 3rd day of the inoculation, reached the maximum on the 5th day and decayed gradually. However, development of lesions in rabbits was irregular and less distinct than that in guinea pigs, and some animals (two out of 11) failed to develop characteristic lesions even at very high doses of the pathogen. Therefore, it was not always easy to determine the end point in terms of the highest dilution producing the haemorrhagic leison. The end point estimated in rabbits seemed to be considerably lower than that in guinea pigs. The haemorrhage was localized in the dermis and did not ordinarily extend to the under layer of the skin.

\section{Agglutinin Production in Rabbits and Guinea Pigs after Infection}

The difference in the pattern of development of local haemorrhage between rabbits and guinea pigs may suggest that the recovery is related to the production of the specific antibody. An attempt was therefore made to compare the antibody production after infection of both species. Four rabbits weighing about $3 \mathrm{~kg}$ were inoculated intracutaneously as stated above and bled daily by carciac puncture. Fifteen guinea pigs of 450 to $500 \mathrm{~g}$ were used for this experiment, as larger animals were more suitable for long-term observation due to their higher resistance against the challenge than smaller ones, as will be mentioned below. Of 15 guinea pigs, only five survived for the observation period of 21 days. As shown in Fig. 2, agglutinin production in rabbits occurred apparently earlier than in guinea pigs. In the former, agglutinin had been produced before haemorrhage at the inoculated site reached the maximum, whereas in the latter agglutinating antibody was usually detected later

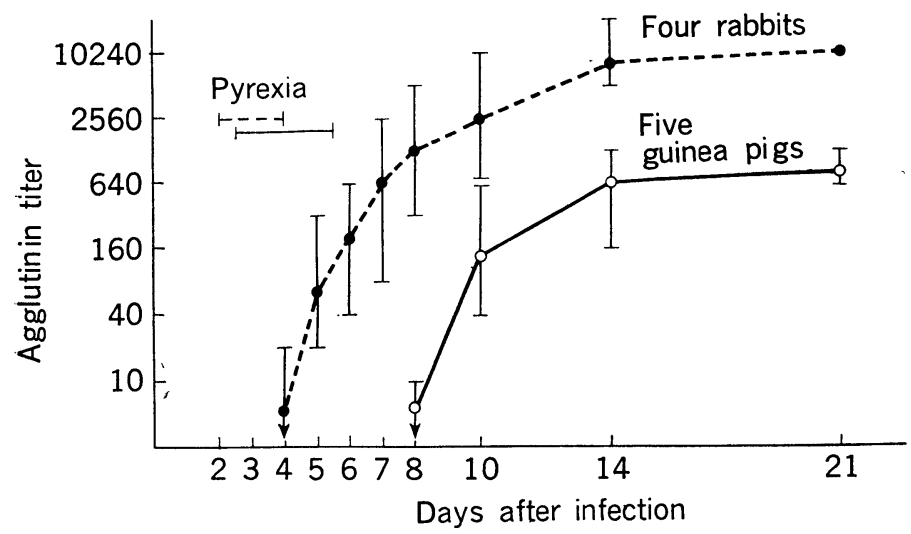

Fig. 2. Courses of agglutinin production in rabbits and guinea pigs infected with $L$. icterohaemorrhagiae Shibaura strain.

The geometric mean agglutinin titers are plotted with respect to each species of animals. The distribution of the titers for each point is shown by a vertical bar. 
than the accomplishment of the haemorrhage.

\section{Effects of Body Weight of the Guinea Pig on Development of Local Haemorrhage}

Guinea pigs were exclusively used for the further experiments because of their high susceptibility to the skin infection. However, when guinea pigs of less than $400 \mathrm{~g}$ were inoculated with high doses of the pathogen, a greater part of the animals died before the 7th day. The use of larger animals is preferable as higher doses may often be applied in the actual titration of virulence of the organism of different origins. It was, therefore, desired to determine whether similar patterns of the lesions comparable with those stated above could be produced in larger animals which seemed more resistant to the infection. Thirty guinea pigs were divided into three groups of 10 animals by body weight, $560-630 \mathrm{~g}, 445-480 \mathrm{~g}$, and $315-345 \mathrm{~g}$. Each animal of the three groups was injected intracutaneously with serial dilutions, $10^{-3.5}$ to $10^{-8}$, of the leptospiral suspension at intervals of 0.5 in logarithm. In addition, each of five animals of each group received another dose of a $10^{-1}$ dilution (Table I). Body weights of the animals had no effects on the end point in the cases surviving more than 7 days after the inoculation. In the guinea pigs inoculated with the additional dose, the end point was not determined accurately because all the animals died within 6 days. However, the inoculation of the additional dose did not seem to have influenced the development of haemorrhage at the sites inoculated with higher dilutions. In another experiment such a high dose as 100 MLD was injected intraperitoneally 3 days before the ic infection. The pretreatment did not influence the development of

\section{TABLE I}

Effects of the body weight of the guinea pig and the additional injection with a higher dose of the inoculum on appearance of local haemorrhage

\begin{tabular}{cccccccc}
\hline & & \multicolumn{5}{c}{$\begin{array}{c}\text { The highest dilution (in exponent) inducing } \\
\text { haemorhage at the injection site }\end{array}$} \\
\cline { 3 - 7 } $\begin{array}{c}\text { Group by } \\
\text { body weight }\end{array}$ & $\begin{array}{c}\text { Additional } \\
\text { injection }^{2)}\end{array}$ & \multicolumn{5}{c}{ Days after injection } \\
& & 3 & 4 & 5 & 6 & 7 \\
\hline $560-630 \mathrm{~g}$ & + & -4.3 & -4.8 & -5.3 & $(\mathrm{D})-5.3$ & $/$ \\
& - & -4.1 & -4.8 & -5.0 & -5.6 & -5.6 \\
$445-480 \mathrm{~g}$ & + & -4.4 & -5.6 & -5.6 & $(\mathrm{D})-5.6$ & $/$ \\
& - & -4.2 & -5.1 & -5.5 & -5.5 & -5.5 \\
$315-345 \mathrm{~g}$ & + & -4.3 & -5.0 & $(\mathrm{D})-5.0$ & $/$ & $/$ \\
& - & -4.3 & -4.9 & -5.0 & -5.4 & -5.6 \\
\hline
\end{tabular}

1) Each group consisted of 10 animals.

2) Each animal was injected with serial dilutions, $10^{-3.5}$ to $10^{-8.0}$, of the leptospiral suspension. Each of five animals of the group received an additional injection with a $10^{-1}$ dilution of the leptospiral suspension.

3) Geometric mean of the highest dilution of the inoculum showing local haemorrhage was shown.

(D) shows death of all the animals on the date indicated. No difficulty was encountered to determine the end point of haemorrhage of dead animals. 
local haemorrhage, and the end points observed in a few survivors were comparable with those obtained in the animals without the pretreatment of ip inoculation. It may, therefore, be favorable to use larger guinea pigs which can survive the infection with higher doses for routine titration of the virulence.

\section{Determination of $50 \%$ Infective Dose $\left(I D_{50}\right)$ and Minimum Infective Dose (MID) by the Intracutaneous Method}

The time course of development of local haemorrhage was pursued precisely. One-tenth milliliter of each of serial dilutions of the leptospiral suspension from $10^{-2}$ to $10^{-8}$ was injected into the skin of each of three animals. Rectal temperature and diameter of local lesion were measured daily. Figure 3 shows three typical patterns. of development of local haemorrhage in guinea pigs. All the animals had fever over $40 \mathrm{C}$ on either the $3 \mathrm{rd}$ or the 4 th day. Local haemorrhage became visible on the 2nd or the 3rd day with the lowest dilutions of the pathogen. The incubation period for the local haemorrhage was usually a little shorter than that for the general symptom, pyrexia. Haemorrhage proceeded until the 7 th day of the inoculation, and end point no longer changed usually after the 8th day. Therefore, the end point was. determined usually on the 7 th day.

To determine $\mathrm{ID}_{50}$ of the leptospiral suspension, each of at least five guinea pigs of about $450 \mathrm{~g}$ by weight was injected intracutaneously with a series of dilutions of

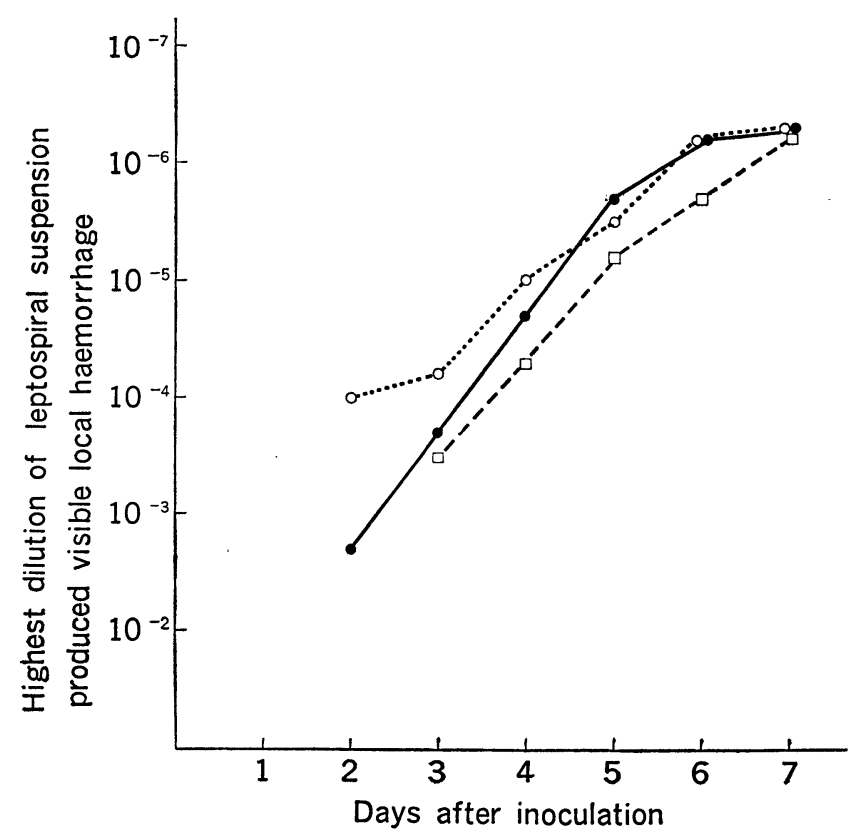

Fig. 3. Time courses of visible local haemorrhage after inoculation (three experiments).

One-tenth $\mathrm{ml}$ each of serial dilutions was injected into the skin of three guinea pigs in each experiment. The animals were observed daily for appearance of local haemorrhage.

Each point shows the geometric mean of the highest dilution of leptospiral suspensions producing visible haemorrhage on the date indicated. 
the leptospiral suspension from $10^{-4.0}$ to $10^{-9.5}$ at intervals of logarithmic 0.5. Figure 4 shows the dose response curves obtained in five experiments. A certain correlation

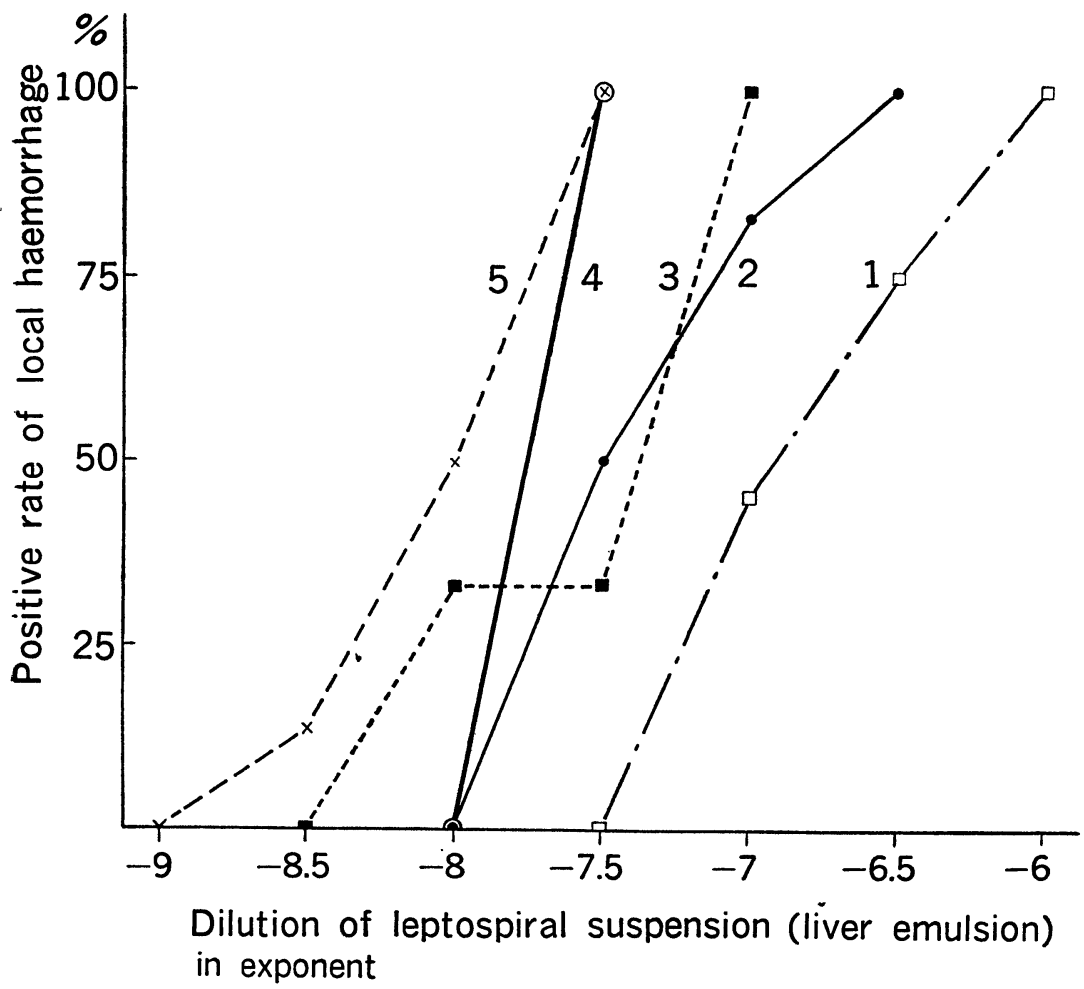

Fig. 4. Dose-response curves of localized haemorrhage (five experiments).

Curve Nos. correspond to liver Nos. in Table II.

TABLE II

Number of leptospira per $I D_{50}$ of the leptospiral suspension ${ }^{1)}$

\begin{tabular}{cccc}
\hline Liver ${ }^{3)}$ & $\begin{array}{c}\text { No. of leptospira } \\
\text { per gram of liver } \\
\left(\times 10^{8}\right)^{3)}\end{array}$ & $\begin{array}{c}\text { ID }_{50} \text { per gram } \\
\text { of liver } \\
\text { (in exponent) }\end{array}$ & $\begin{array}{c}\text { Leptospira } \\
\text { per ID }\end{array}$ \\
\hline 1 & 2.8 & $6.9(20)$ & 34.6 \\
2 & 6.8 & $7.5(6)$ & 21.5 \\
3 & 3.8 & $7.4(6)$ & 15.8 \\
4 & 3.7 & $7.8(5)$ & 6.3 \\
5 & 6.1 & $8.1(6)$ & 6.1 \\
\hline
\end{tabular}

1) A $10 \%$ emulsion of the liver of a guinea pig dying from infection with Shibaura strain was allowed to stand for $5 \mathrm{~min}$; the supernatant fluid was used.

2) The liver Nos. correspond to those of curves in Fig. 4.

3) The organisms were counted with a Petroff-Hausser's bacterial counter.

4) The number of guinea pigs used for determining $\mathrm{ID}_{50}$ is shown in parentheses. 
is observed in each experiment between the rate of positive lesions and the doses of the pathogen. Therefore, $\mathrm{ID}_{50}$ of the inocula were calculated by Reed and Münch's method. Leptospires in the livers from which the leptospiral suspensions were prepared were counted; the number of the organisms per $\mathrm{ID}_{50}$ of each liver was also determined. The results are summarized in Table II. $\mathrm{ID}_{50}$ thus determined contained from 6 to 35 organisms. It seems rather practical, however, to determine MID instead of $\mathrm{ID}_{50}$ by a rough titration of the virulence of the leptospiral suspension with a small number of animals. For convenience, the authors propose that the highest dilution developing apparent local haemorrhage in more than half of the animals shall be taken as MID in $0.1 \mathrm{ml}$. For this purpose, dilution of the suspension should be carried out at intervals of $0.25-0.5$ in logarithm.

\section{Comparative Study on the Course of the Disease in Guinea Pigs Inoculated Intracutaneously or Intraperitoneally}

Eighty guinea pigs of 400 to $430 \mathrm{~g}$ were divided into 16 groups. Eight groups were inoculated intracutaneously and the remaining eight groups intraperitoneally as shown below. Dilutions of the leptospiral suspension were so prepared as to contain

TABLE III

Appearance of the symptoms in guinea pigs inoculated intraperitoneally or intracutaneously with serially diluted leptospiral suspension

\begin{tabular}{|c|c|c|c|c|c|c|}
\hline \multirow{2}{*}{$\begin{array}{l}\text { No. of leptospira } \\
\text { in injection dose } \\
\text { (in exponent) }\end{array}$} & \multirow{2}{*}{$\begin{array}{l}\text { Inoculation } \\
\text { route }\end{array}$} & \multicolumn{5}{|c|}{ Onset time in day of the symptoms ${ }^{1)}$} \\
\hline & & $\begin{array}{c}\text { Local } \\
\text { haemorrhage }\end{array}$ & Pyrexia & Jaundice & Death & Survivors ${ }^{3)}$ \\
\hline \multirow[t]{2}{*}{6.5} & ip & & $1.6(1-2)$ & $3.6(3-5)$ & $5.0(4-6)$ & $0 / 5$ \\
\hline & ic & $1.6(1-2)$ & $1.8(1-2)$ & $5.6(5-6)$ & $6.0(5-7)$ & $0 / 5$ \\
\hline \multirow[t]{2}{*}{4.5} & ip & & $3.4(3-4)$ & $5.8(5-7)$ & $6.8(5-9)$ & $0 / 5$ \\
\hline & ic & 2.0 & $3.4(3-4)$ & $5.8(5-7)$ & $7.6(7-9)$ & $0 / 5$ \\
\hline \multirow[t]{2}{*}{2.5} & ip & & $4.0(3-5)$ & $7.2(6-8)$ & $8.8(8-10)$ & $0 / 5$ \\
\hline & ic & $3.8(3-4)$ & 5.0 & $7.4(6-9)$ & $8.8(8-9)$ & $0 / 5$ \\
\hline \multirow[t]{2}{*}{2.0} & ip & & $4.2(4-5)^{2)}$ & $7.2(6-8)^{2)}$ & $8.5(8-9)^{2)}$ & $1 / 5$ \\
\hline & ic & $4.4(4-5)$ & 5.0 & $8.2(7-9)$ & 9.0 & $0 / 5$ \\
\hline \multirow[t]{2}{*}{1.5} & ip & & $6.0^{2)}$ & $8.5(8-9)^{2)}$ & $9.0^{2)}$ & $3 / 5$ \\
\hline & ic & 5.0 & $5.2(5-6)$ & $8.6(8-10)$ & $9.6(9-11)$ & $0 / 5$ \\
\hline \multirow[t]{2}{*}{1.0} & ip & & $6.0^{2)}$ & $8.0(7-9)^{2)}$ & $8.5(8-9)^{2)}$ & $1 / 5$ \\
\hline & ic & 6.0 & 6.0 & $8.8(8-10)$ & $10.0(9-11)$ & $0 / 5$ \\
\hline \multirow[t]{2}{*}{0.5} & ip & & - & - & - & $5 / 5$ \\
\hline & ic & - & - & - & - & $5 / 5$ \\
\hline \multirow[t]{2}{*}{0} & ip & & - & - & - & $5 / 5$ \\
\hline & ic & - & - & - & - & $5 / 5$ \\
\hline
\end{tabular}

(1) Onset time of the symptoms are presented in an arithmetic mean; the minimum and the maximum are shown in parentheses.

.2) Some animals showed no symptoms during the observation period. Such animals were excluded from the calculation of the mean.

.3) Number of survived animals/Number of animals used. 
$10^{6.5}, 10^{4.5}, 10^{2.5}, 10^{2.0}, 10^{1.5}, 10^{1.0}, 10^{0.5}$ and $10^{\circ}$ organisms in an injection volume of $0.1 \mathrm{ml}$ and $1.0 \mathrm{ml}$ for ic and ip infection, respectively. Only one dilution was used for each animal. All animals were observed for 12 days. The results are summarized in Table III.

The courses of the diseases caused by the ic and ip routes were similar, but the lag period was a little longer in the former cases. Although the survival time of the ic groups seemed longer than that of the ip groups, there was little difference between the two groups in the smallest number of leptospires required to cause death. All 30 animals inoculated with 10 or more leptospires all were killed in the ic groups, whereas five survived in the ip groups. Such difference between the two methods. was found among the animals injected with such lower doses as $10^{1.0}-10^{2.0}$ of leptospires. All the survived animals were killed by a subsequent challenge with a more highly virulent leptospiral suspension. The fact may suggest that infection was not established in those survivors by the first inoculation.

\section{Histological Changes at the Inoculated Site}

The inoculated site of the skin showing typical haemorrhage was taken for microscopy 7 days after infection. In general main histological changes were observed in the dermis and epidermis covering the inoculated area. Thickening of the epidermis in various grades was found (Fig. 5). Haemorrhage was found mainly in the dermis, particularly just under the epidermis. Proliferation of capillary endothel and infiltration of neutrophilic leucccytes was also observed. Phagocytosis of leptospires. by the leucccytes was found there. Thickening of interstitium of the subcutaneous. fatty tissue with marked mononuclear cell proliferation was apparent, and single cell degeneration of the muscle tissue was also recognized (Fig. 6). The draining lymphnodes were swollen and haemorrhagic, and phagocytosis of leptospires by macrophages and reticulum cells was frequently found in it (Fig. 7).

A considerablly large number of leptospires were demonstrated in the thickened epidermal layer as well as in the epidermic hair-follicle (Fig. 8). Not a few pathogens were observed even when infected with only several to several tens of the pathogen, nearly MID dose. These facts suggest that the leptospires may have proliferated at the place: A group of the pathogen in the epidermic hair-follicle is shown in Fig. 9.

Fig. 1. Local haemorrhage in a guinea pig produced at the inoculated sites 7 days after ic infection.

Fig. 5. Thickening of the epidermis and haemorrhage in the dermis at the inoculated site. $(\mathrm{H}-\mathrm{E} \times 120)$

Fig. 6. Thickening of interstitium of the subcutaneous fatty tissue with marked mononuclear cell proliferation and single cell degeneration of the muscle just under the inoculated site. The arrow shows muscle cell degeneration. (H-E $\times 120)$

Fig. 7. Haemorrhage and phagocytosis of leptcspires by reticulum cells in the draining lymph node. (Levaditi's method $\times 600$ )

Fig. 8. Leptospires in the thickened epidermal layer at the inoculated site. (Levaditi's method $\times 600$ )

Fig. 9. Leptospires in a hair-follicle at the site inoculated with only several organisms.

(Levaditi's method $\times 600$ ) 


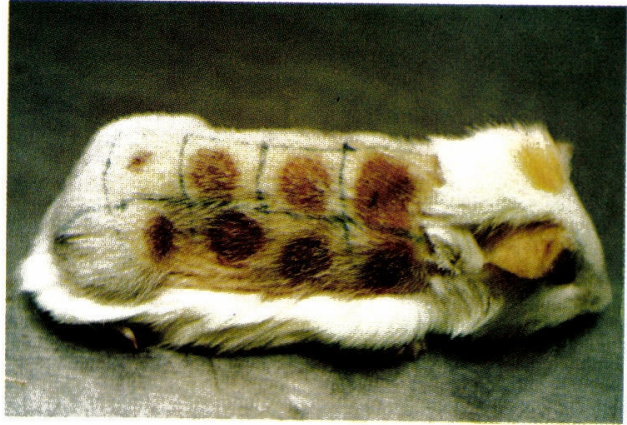

Fig. 1

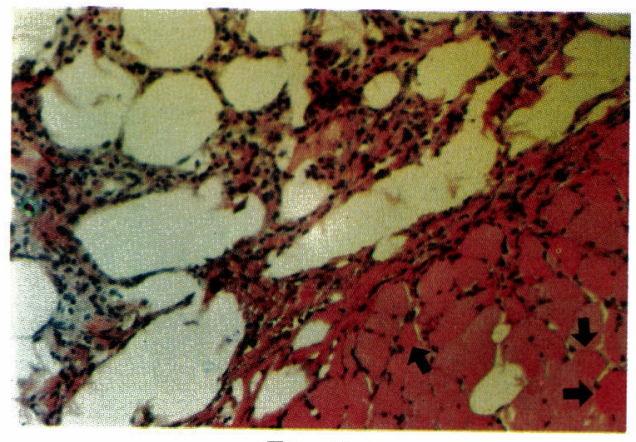

Fig. 6

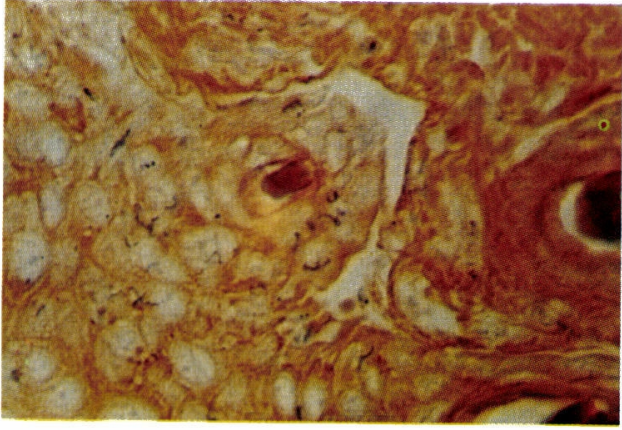

Fig. 8

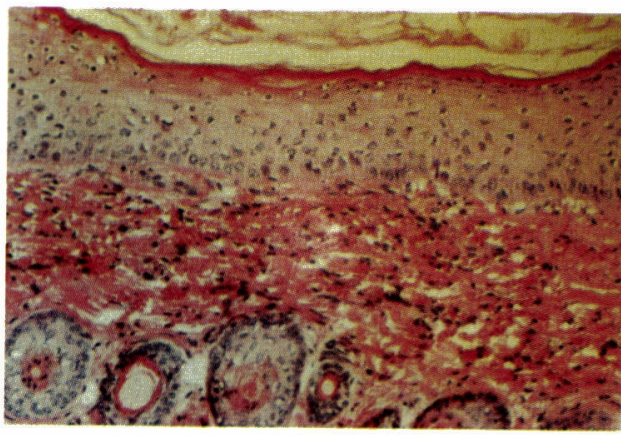

Fig. 5

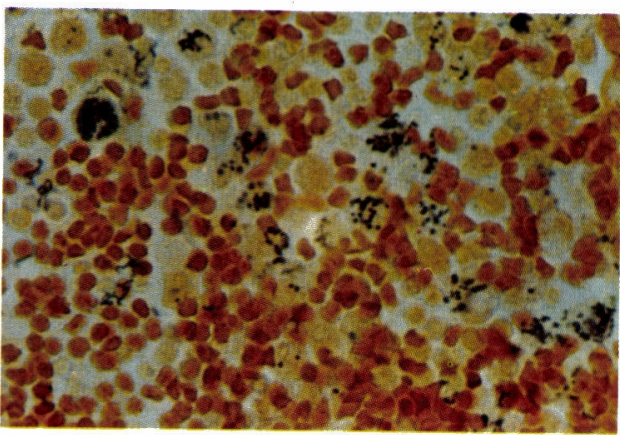

Fig. 7

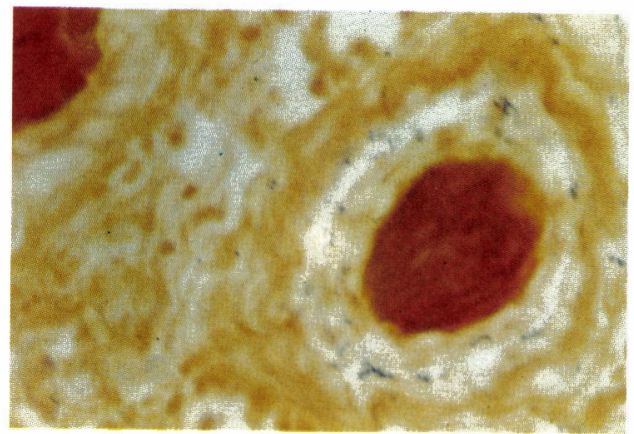

Fig. 9

\section{Prevention of the Local Haemorrhage in Actively or Passively Immunized Guinea Pigs}

Two experiments were carried out to know whether or not development of local haemorrhage could be prevented in the immunized animałs. The effect of active immunization was first investigated. Ten guinea pigs weighing about $250 \mathrm{~g}$ were divided into two groups. Five animals were immunized twice at an interval of 1 week with a phenolized vaccine in doses of 500 million leptospires. The other group served as 
the control without vaccination. A challenge with $10^{4} \mathrm{MID}$ was given intracutaneously 2 weeks after the last immunization. No sign of haemorrhage was observed in the immunized animals, whereas typical local haemorrhage was produced in the control ones. The effect of passive immunization was then examined. Six guinea pigs weighing about $450 \mathrm{~g}$ were divided into three groups. One group received $1 \mathrm{ml}$ of undiluted antiserum obtained from the infected guinea pigs 6 weeks after the infection; another a 100 -fold dilution of the antiserum, and the last one served as the control without pretreatment. After $24 \mathrm{hr}$, each of the animals was inoculated intracutaneously with $0.1 \mathrm{ml}$ portion of serial dilutions of the leptospiral suspension at intervals of 0.5 in logarithm, covering a range of $10^{0.5}$ to $10^{7.0}$ organisms per ml. The results are shown in Table IV. No haemorrhage was produced in the animals treated with the undiluted

TABLE IV

Effect of passive immunization on appearance of local haemorrhage

\begin{tabular}{|c|c|c|c|c|c|c|}
\hline \multirow{3}{*}{$\begin{array}{l}\text { Serum } \\
\text { dilution }\end{array}$} & \multicolumn{6}{|c|}{$\begin{array}{l}\text { The minimum number of the pathogen producing } \\
\text { local haemorrhage (in exponent) }\end{array}$} \\
\hline & \multicolumn{6}{|c|}{ Days after infection } \\
\hline & 2 & 3 & 4 & 5 & 6 & 7 \\
\hline \multirow[t]{2}{*}{$10^{0}$} & - & - & - & - & - & - \\
\hline & - & - & - & - & - & - \\
\hline \multirow[t]{2}{*}{$10^{-2}$} & - & 6.0 & 5.5 & 4.0 & 4.0 & 4.0 \\
\hline & - & 5.0 & 5.0 & 4.5 & 2.5 & 2.5 \\
\hline - & - & 5.5 & 4.5 & 3.0 & 1.5 & 1.5 \\
\hline (Control) & - & 5.5 & 5.0 & 2.5 & 0.5 & 0.5 \\
\hline
\end{tabular}

Passive immunization was given $24 \mathrm{hr}$ before the challenge with serial dilutions of the leptospiral suspension.

serum, whereas haemorrhage appeared on the 3rd day in the control group and progressed rapidly. In the animals having received a $10^{-2}$ dilution of the serum, haemorrhage developed on the 3rd day but it was weaker than that of the control group. The end point of local haemorrhage in the animals having received $10^{-2}$ serum apparently differed from that in the control group. Besides, no jaundice was observed in the $10^{-2}$ serum group in contrast with the control animals which showed severe jaundice.

\section{Discussion}

Experimental leptospirosis was successfully induced in guinea pigs by ic infection with L. icterohaemorrhagiae Shibaura strain. When the leptospiral suspension was injected into the skin of guinea pigs, apparent haemorrhage was produced at the injected site a few days after the inoculation before or on the day of onset of pyrexia. The development of the local haemorrhage was studied with special reference to the doses of the organisms. The larger the dose, the earlier the appearance of local haemorrhage. All the animals producing local lesions were invariably accompanied with severe 
general symptoms. Once local haemorrhage developed, the disease advanced in similar patterns regardless the inoculum size. The development of the local haemorrhage with a smaller inoculum was not affected by either simultaneous or previous infection with a larger inoculum.

The presence of a large number of the pathogens within the epidermis of the inoculation site shows that it has no connection with extravasation because of the absence of capillary vessels there. The pathogen seems to have a high affinity for the epidermis tissue. Local haemorrhage might rather be connected with proliferation of the pathogen at the inoculated site and occur independently from general symptoms. Alternatively, the organisms may accumulate secondarily as the result of extravasation, since histological examinations also revealed that the presence of the pathogen in other layers appears to be more or less accompanied with haemorrhage. It is interesting to know how distinctly localized haemorrhage appears at the inoculated site. Since haemorrhage in the skin is also produced as one of the general symptoms not only in clinical cases but also in animals infected experimentally through the ip route, a comparative examination on the general and local haemorrhage may give a clue to understand the mechanism of haemorrhage in this disease. The problems will be treated in future investigation.

Smaller guinea pigs were inadequate to titrate the virulence by the ic method since they died before development of the local lesion at lower doses; we usually use animals of more than $450 \mathrm{~g}$ for titration of the virulence. The rabbit was not suitable for titration because of the early disappearance of local haemorrhage. It might possibly be due to the earlier production of the antibodies, although the agglutinin itself may not necessarily be leptospiricidal or protective antibodies. On the contrary, the guinea pig may die from severe disease before the antibody reaches the level to save animals.

Another experiment showed a considerable variation in the virulence of the leptospiral suspensions prepared from the livers of the dying guinea pigs inoculated intraperitoneally (unpublished data). In addition, animals inoculated intraperitoneally are more liable to escape from infection than those inoculated intracutaneously, especially at lower doses (Table III). Fate of the pathogen inoculated intraperitoneally seems to be irregular as compared with those inoculated intracutaneously. The ic method is useful for titrating the virulence of the pathogen, because the MID determined by the method was almost comparable with that by the ip method. The method may be of use to save animals and labour for the titration.

The number of leptospira per $\mathrm{ID}_{50}$ was several to several tens in accordance with the results by Yanagawa et al. (1959). They demonstrated that the numbers of the organism per $\mathrm{ID}_{50}$ in mice were 11.2 and 1.9 for a strain of L. icterohaemorrhagiae and that of L. autumnalis, respectively. On the other hand, using leptospira of another serotype we experienced that $10^{-3}$ or more organisms were required to produce infection in guinea pigs (unpublished data).

Local haemorrhage was protected completely by not only active but also passive immunization. The fact suggests a possibility of applying the intracutaneous method for assay of potencies of both the therapeutic antiserum and the vaccine. Studies on the former will be reported in a separate paper.

As shown in the experiments using rabbits, susceptibility to the same pathogen may differ depending on the animal species, and the course of infection in different 
animal species may also vary depending on the strain or serotype of leptospira. As generally well known, the mouse usually shows no sign of infection with various. serotypes of leptospira, but very often becomes a reservoir of leptospires. Furthermore, apparent local haemorrhage as shown in this work is not necessarily produced in the guinea pig by any virulent strain of L. icterohaemorrhagiae tested (unpublished data). This suggests that the mechanism of local haemorrhage might differ from that of general haemorrhage. Further experiments on these problems should be made tos elucidate the mechanisms of infection with leptospira.

\section{ACKNOWLEDGEMENT}

The authors wish to thank Dr. Yasuyuki Egashira, Director of Department of Pathology, National Institute of Health, for his kind consultation regarding the problems in histopathology. They also wish to express their thanks to Dr. Ryosuke Murata, Director of the 2nd Department of Bacteriology, National Institute of Health, for his helpful advice and encouragement in performing the present investigation.

This work was partly supported by a grant for Scientific Research by Ministry of Health. and Welfare.

\section{REFERENCES}

INADA, R. (1951): Leptospirosis icterohaemorrhagica (Weil's disease). Nihon Isho Shuppan Ltd., Tokyo (in Japanese). $181 \mathrm{p}$.

KANEKO, R. AND OKUDA, K. (1915): Observations on the lymph nodes in patients at early stage of Japanese icterohaemorrhagic spirochetosis (Weil's disease), especially on the presenceof the spirochaetes. Nihon Iji Shinshi, (No. 1943), 2222-2336 (in Japanese).

SCHÜFFNER, W. AND MOCHTAR, A. (1927): Versuche zur Aufteilung von Leptospiren-Stämmen, mit einleitenden Bemerkungen über Verlaut von Agglutination und Lysis. Zentralbl. Bakteriol., 1. Orig., 101, 405-413.

Yanagawa, R., Hiramune, T., Fujita, J. And Ishil, S. (1959): Experimental studies on infection and immunity in leptospirosis. III. The use of mice for estimation of Leptospirae. Jap. J. Vet. Sci., 21, 259-264. 